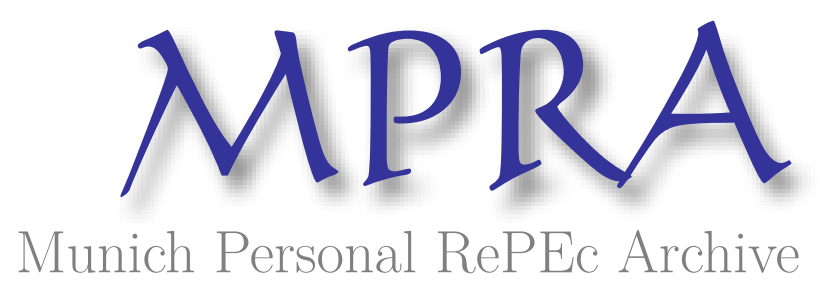

\title{
Estimating Structural Change with Smooth Transition Regressions: an Application to Meat Demand
}

Holt, Matthew T. and Balagtas, Joseph V.

Purdue University

20 May 2009

Online at https://mpra.ub.uni-muenchen.de/15331/

MPRA Paper No. 15331, posted 21 May 2009 13:58 UTC 


\title{
Estimating Structural Change with Smooth Transition Regressions: an Application to Meat Demand
}

\author{
Matthew T. Holt and Joseph V. Balagtas*
}

May 20, 2009

An area of recurring interest in empirical studies of economic behavior based on time series data is the notion of structural change. How, for example, does that analyst know if the parameters embedded in the data generating process have changed over time and, if they have changed, for which data point or points did this change most likely occur? Obtaining suitable answers to these and related questions is often a nontrivial exercise and, moreover, the implications of ignoring structural change altogether can potentially lead to false inference and, by extension, misguided policy recommendations. Of course economists have long recognized the potentially critical role of structural change in data analysis. For example, the so-called Chow test, introduced in a seminal work by Chow (1960), is designed to test for exactly this feature of the data in the context of a linear econometric model, albeit in one with a single, known structural change. Refinements to the Chow test that allow for, among other things, testing when the change point is unknown, have been advanced in recent years by Andrews (1993), Andrews and Ploberger (1994), and Hansen (1996), among others.

In the agricultural economics literature there has been considerable focus over time on structural change in the demand for food and, most notably, in the demand for meat. Examples pertaining especially to meat demand in the U.S. and elsewhere include Chavas (1983), Dahlgran (1987), Eales and Unnevehr (1988) Moschini and Mielke (1989), Alston and Chal- 
fant (1991), and more recently, Davis (1997) and Bryant and Davis (2008). Work in a similar spirit has been done in the context of the demand for fats and oils by Goodwin et al. (2003) and in factor demand equations for U.S. food and kindred products manufacturing sector by Goodwin and Brester (1995).

In terms of meat demand, while the methods vary and the causes attributed to the underlying structural change may vary (e.g., increased dietary concerns associated with consuming red meat, etc.) nearly all of the aforementioned studies find support for some form of structural change in meat consumption. In many of these studies the structural change is assumed to be a discrete, one-time event. In several instances the possibility of a smooth structural change over time is allowed. See, for example, Moschini and Mielke (1989). But to the best of our knowledge missing from the extant literature is a model that permits the possibility that (1) structural change is smooth over time and (2) that it is potentially non-monotonic. In this paper we explore exactly these possibilities in the context of U.S. meat demand relationships. In so doing we follow closely the testing and estimation strategy for univariate time-varying regression models put forward by Lin and Teräsvirta (1994), which in turn builds upon related work on smooth transition regressions by Terasvirta (1994). ${ }^{1}$ In the remainder of the paper we discuss the modelling approach and the methods used for testing for potentially smooth structural change. We then present an application to a system of inverse demand equations for meats in the United States.

\section{The IAIDS Model and Structural Change}

The fundamental modelling framework employed here is a system of inverse demand equations applied to meat consumption and price data. In an inverse demand system normalized prices adjust to exogenous changes in quantities, that is, short-run supplies are assumed to be perfectly inelastic. Inverse demand models are useful tools for examining consumer behavior when the consumption goods in question are highly perishable or when they have 
a reasonably short shelf-life (Barten and Bettendorf, 1989). In recent years numerous studies have used inverse demand systems to study aggregate meat demand behavior, including Eales and Unnevehr (1994), Holt and Goodwin (1996), and Holt (2002).

The basic demand system we focus on here is the Inverse Almost Ideal Demand System (IAIDS) model, introduced originally by Eales and Unnevehr (1994). The IAIDS is developed from a particular distance (utility) function, and is parallel in its specification to Deaton and Muellbauer's (1980) Almost Ideal Demand System. The central difference is that in the IAIDS normalized prices are treated as endogenous while quantities are exogenous. In the case of the IAIDS the distance function is of the form

$$
\ln D(q, u)=(1-u) \ln a(q)+u \ln b(q)
$$

where $q$ is a $(n x 1)$ vector of quantities, $u$ denotes the utility index, $a(q)$ is a translog quantity index given by

$$
\ln a(q)=\sum_{k} \alpha_{k} \ln \left(q_{k}\right)+\frac{1}{2} \sum_{k} \sum_{j} \gamma_{k j}^{*} \ln q_{k} \ln q_{j}
$$

and

$$
\ln b(q)=\ln a(q)+\beta_{0} \prod_{k} q_{k}^{-\beta_{k}}
$$

In $(2)$ and $(3), k, j=1, \ldots, n$.

Application of the Shephard-Hannoch Lemma through differentiation of the logarithmic distance function with respect to (logarithmic) quantities gives expenditure share equations for each good as a function of quantities and the utility index. The share equations can be "uncompensated" to remove utility by recognizing that at the optimum $\ln D(q, u)=0$, thereby implying that (1) can be inverted and solved for $u$ as a function of $q$, the direct utility function $u(q)$. Then, $u(q)$ may be used to substitute for $u$ in each share equation. 
Doing so in the present case yields share equations of the form:

$$
w_{i}=\alpha_{i}+\sum_{j} \gamma_{i j} \ln q_{j}+\beta_{i} \ln (Q)
$$

where $w_{i}=\frac{p_{i} q_{i}}{y}$, and where $p_{i} / y$ denotes the nominal price for the $i^{\text {th }}$ good divided by group expenditure, $y$, and where $i=1, \ldots, n$. The quantity index $\ln Q$ is defined by

$$
\ln Q=\alpha_{0}+\sum_{k} \alpha_{k} \ln \left(q_{k}\right)+\frac{1}{2} \sum_{k} \sum_{j} \gamma_{k j} \ln q_{k} \ln q_{j}
$$

and $\gamma_{i j}=\frac{1}{2}\left(\gamma_{i j}^{*}+\gamma_{j i}^{*}\right)$. As well, we follow usual practice and restrict $\alpha_{0}=0$ in all subsequent analyses.

Linear homogeneity of the distance function, symmetry of the second-order derivatives, and adding-up across the share equations implies the following set of equality restrictions:

$$
\sum_{i=1}^{n} \alpha_{i}=1, \quad \sum_{i=1}^{n} \gamma_{i j}=\sum_{j=1}^{n} \gamma_{i j}=0, \quad \sum_{i=1}^{n} \beta_{i}=0, \quad \gamma_{i j}=\gamma_{j i}
$$

As required for a locally flexible functional form, there are $n(n-1) / 2$ free parameters in the Antonelli (Hessian) matrix for the IAIDS model. Formulae for computing own, cross-price, and scale flexibilities from the IAIDS are provided by Eales and Unnevehr (1994).

To make the IAIDS empirically meaningful, it is necessary to add stochastic terms to the share equations in (4). Doing so yields a share equation system of the form

$$
w_{i t}=f\left(q_{t}, \theta\right)+e_{i t}=\alpha_{i}+\sum_{j} \gamma_{i j} \ln q_{j t}+\beta_{i} \ln \left(Q_{t}\right)+e_{i t}
$$

where $e_{i t}$ 's are mean zero error terms that are assumed to be joint-normally distributed and where a time subscript, $t, t=1, \ldots, T$, has been added in recognition of the fact that time-series data are to be employed. As well, in (7) $\theta$ is a vector of unknown parameters to be estimated, and includes the $\alpha_{i}, \gamma_{i j}$, and $\beta_{i}$ parameters. ${ }^{2}$ If seasonal data are used the 
model in (7) may be modified to allow the $\alpha_{i}$ parameters to shift by season, which in turn is typically accomplished by incorporating seasonal dummy variables.

The modelling framework outlined thus far is suitable if there is no reason to suspect that structural change has occurred, that is, if there is no reason to believe that one or more of the elements in $\theta$ has changed over the sample period. As a practical matter this is often not the case, especially if the sample covers a substantive time span. In short, changes in habits, tastes and preferences, product quality, product packaging and processing, and so forth are not directly observed but will often have a substantive impact on consumer choices. As noted in the introduction, this is generally posited to be the case for meat demands in the United States over the post-war period. The system in (7) can be modified to allow for structural change. For example, the system could simply be expressed as

$$
w_{i t}=f\left(q_{t}, \theta^{*}\right)+e_{i t},
$$

where $\theta^{*}=\theta_{1}+\theta_{2} D(t)$, and where $D(t)$ is a variable that indicates structural change. For example, if structural change is thought to be a discrete, one-time event occurring at time $\tilde{t}$, then $D(t)$ could be specified to equal one if $t>\tilde{t}$, and zero otherwise. In this case (8) effectively becomes a multivariate switching regression model. Alternatively, if structural change is thought to be continuous (but linear), then we might specify $D(t)=t$, an approach advocated by, for example, Farley et al. (1975). Moreover, the approach is flexible in that a some subset of the parameters in $\theta_{1}$ could be held constant while select others could be allowed to vary (e.g., the constant terms). Both approaches have been utilized extensively in the investigation of structural change in meat demand.

A slight variant of the model in (8) may be expressed as follows:

$$
w_{i t}=f\left(q_{t}, \theta_{1}\right)\left(1-G\left(t^{*} ; \gamma, c\right)\right)+f\left(q_{t}, \theta_{2}\right) G\left(t^{*} ; \gamma, c\right)+e_{i t}
$$

where $t^{*}=t / T, G($.$) , the transition function, is a possibly smooth and continuous function of$ 
$t^{*}$ that is bounded on the unit interval, and $\gamma$ and $c$ are parameters which define the nature of the transition function. The model in (9) is a parallel to the time-varying regression models considered in a univariate context by Lin and Teräsvirta (1994). Indeed, the model in (9) can be thought of as a slight variation of both the switching regression and continuous linear change models implied by (8). Of course the choice of the transition function is central to the analysis.

Several candidates exist for defining $G\left(t^{*} ; \gamma, c\right)$. A common specification for the transition function in (9) is the first-order logistic function, given by

$$
G\left(t^{*} ; \gamma, c\right)=\left[1+\exp \left\{-\gamma\left(t^{*}-c\right) / \sigma_{t^{*}}\right\}\right]^{-1}, \gamma>0
$$

where $\gamma$ is the speed-of-adjustment parameter that determines how quickly the model shifts from one regime to another, $c$ is the centrality parameter that determines at what point in the sample the structural change is $50-$ percent complete, and $\sigma_{t^{*}}$ is the standard deviation of the normalized trend variable. Dividing $\gamma$ by $\sigma_{t^{*}}$ renders the speed-of-adjustment parameter unit free. Interestingly, as $\gamma \rightarrow \infty$ the first-order logistic function in (10) becomes a Heaviside indicator function that assumes the value of zero if $t^{*}<c$ and is one otherwise. In other words, in this case the model reduces to the switching regression model. Likewise, as $\gamma \rightarrow 0$ the transition function becomes effectively linear in $t^{*}$. In this manner the model that combines (10) with (9) encompasses all possibilities implied by (8).

Another approach is to specify $G\left(t^{*} ; \gamma, c\right)$ as an exponential function, that is, as

$$
G\left(t^{*} ; \gamma, c\right)=1+\exp \left\{-\gamma\left(t^{*}-c\right)^{2} / \sigma_{t^{*}}^{2}\right\}, \gamma>0
$$

In this case the structural change implied by the transition function in (11) is non-monotonic and is symmetric around $c$. In this case as $t^{*} \rightarrow \pm \infty, G\left(t^{*} ; \gamma, c\right) \rightarrow 1$, whereas $G\left(t^{*} ; \gamma, c\right)=0$ when $t^{*}=c$. Finally, as $\gamma \rightarrow 0$ or $\gamma \rightarrow \infty$, the exponential function approaches zero and one, respectively. Henceforth we refer to the demand system that utilizes the exponential 
model as the second-order model.

A final specification for $G\left(t^{*} ; \gamma, c\right)$ considered by Lin and Teräsvirta (1994) is the thirdorder logistic function

$$
G\left(t^{*} ; \gamma, c_{1}, c_{2}, c_{3}\right)=\left[1+\exp \left\{-\gamma \prod_{i=1}^{3}\left(t^{*}-c_{i}\right) / \sigma_{t^{*}}\right\}\right]^{-1}, c_{1} \leq c_{2} \leq c_{3}, \gamma>0
$$

In the case of (12) the structural change is not necessarily monotonic, and multiple regime switches are possible. In empirical work a useful formulation of (12) is to impose the restriction $c=c_{1}=c_{2}=c_{3}$. In this case structural change is monotonic but plateaus around the value of $c$ are possible. We refer to the demand model that uses the transition in (12) as the third-order model.

While the above specifications for transition functions in a demand system allow considerable flexibility in modelling structural change, there is a need to be able to test for these various alternatives against the null of a demand model with no structural change. But in this instance the standard likelihood ratio test will not have the correct asymptotic size because there are unidentified nuisance parameters, notably $\gamma$ and $c$ in the transition function, under the null hypothesis of "no structural change." The result is that the distribution of the likelihood ratio test statistic will be non-standard (Davies, 1977, 1987). A considerable body of literature has arisen in recent years to address these non-standard testing issues. Luukkonen et al. (1988), for example, propose replacing the transition function with a suitable Taylor series approximation evaluated at the point $\gamma=0$. Standard Lagrange Multiplier (LM) tests may then be employed. This approach works well when the null model is univariate and linear in parameters. Of course in this case the basic IAIDS model is not univariate, nor is it linear in parameters. ${ }^{3}$

The approach we adopt here to testing is similar to that advocated by Skalin (1998) and Seo (2006) in the context of smooth transition and multivariate threshold models, respectively. That is, we construct the standard likelihood ratio (LR) test statistic for a test of the 
relevant structural change model vis-à-vis the basic IAIDS. The empirical distribution for the LR test statistic is then constructed by using a reasonably large number, $B$, of parametric bootstraps of the basic IAIDS model. Both the basic and the structural change models are estimated using the resultant pseudo data. The LR test statistic is then computed for each bootstrap draw. In this manner it is possible to construct the empirical distribution for the LR test statistic, and thereby to obtain an empirical $p$-value. Monte Carlo simulations by both Skalin (1998) and Seo (2006) suggest that this approach to testing holds promise in sample sizes typical of those encountered in many time series applications.

\section{An Application to U.S. Meat Demand}

Quarterly data on consumption and retail prices for beef, pork, chicken, and turkey were collected from various USDA sources for the 1960-2004 period for a total of $n=180$ sample observations. Data prior to 1997 were obtained from various sources described in detail by Holt (2002). Data for pork and beef from 1997 through 2004 were obtained from the online version of the U.S. Department of Agriculture (2006b) Red Meat Yearbook. Likewise, data for chicken and turkey were obtained from the online version of the U.S. Department of Agriculture (2006a) Poultry Yearbook. Here we aggregate the chicken and turkey categories to obtain a single "poultry" category. The retail price for poultry is derived by determining the share-weighted averages for chicken and turkey prices, where the shares are with respect to total expenditures on chicken and turkey.

The basic IAIDS model in (7) is estimated first by adding quarterly dummy variables to the model, that is, by allowing the $\alpha_{i}$ parameters to shift by season. As well, preliminary results indicated substantial evidence of first-order autocorrelation in the model's error terms. For this reason the parametric vector autocorrelation correction methods outlined in Holt (1998) were used in all subsequent estimations. ${ }^{4}$ Summary measures of model fit and performance for the basic IAIDS model are reported in Table 1. Among other things, the 
results reveal that even the basic IAIDS model fits the data reasonably well, as the system $R^{2}$ is near one. That said, the curvature conditions (i.e., having the Antonelli matrix be negative semi-definite) are violated at 52 of the data points. All of these violations occur for various sample points in the 1960-1983 period.

The next step is to estimate structural change models that rely on, respectively, the three transition functions identified in (10), (11), and (12), and then to test to determine if these models yield a significant improvement in fit. Preliminary results revealed that the restricted version of (12) provided effectively the same fit to the data as the unrestricted model, and therefore all results for the third-order model are for the case where the restriction $c_{1}=c_{2}=c_{3}$ has been imposed. Again, summary measures of fit along with several key parameter estimates - specifically, the estimates for the $\gamma$ and $c$ parameters in the respective transition functions - for each of the structural change models are presented in Table 1.

Do any of the structural change models represent a statistically significant improvement in fit vis-à-vis the basic IAIDS model? Several measures are used to address this question. First, the system Akaike's information criterion (AIC) values are smaller for each of the structural change models than for the basic IAIDS, indicating that the structural change models are valid (Table 1). Second, the ratio of the determinant of the residual covariance matrix for the structural change models relative to that for the basic IAIDS model ranges between $70-$ and $73-$ percent, again indicating that allowing for structural change facilitates a substantial improvement in fit. As well, results reported in Table 1 that there is a considerable reduction in curvature violations when structural change of the sort considered here is allowed for, with several specifications (, i.e., the first- and third-order models) being associated with no violations. Finally, we perform a statistical test of the validity of each structural change model relative to the basic IAIDS. To do so we employed a dynamic parametric bootstrap - wherein a multivariate normal distribution is used - of the basic IAIDS model to generate $B=999$ pseudo data sets, each consisting of $n=180$ sample observations. Each of these pseudo data sets is then used in turn to (1) re-estimate the basic IAIDS model, 
and (2) to estimate each of the three structural change IAIDS models being considered here. For each of these models the LR test statistic is then constructed for each pseudo sample. In this manner it is possible to construct the empirical distribution of the LR test statistic. In all cases, and as reported in Table 1, we find that the basic IAIDS model without structural change is rejected at the 0.001 level in favor of the relevant structural change model. These results then provide substantive evidence that structural change is a feature of meat demands in the United States, a conclusion that is, moreover, supported by considerable prior research.

Among the three structural change models, results recorded in Table 1 suggest that the one that uses the exponential transition function fits the meat data best overall as measured by any of the measures of fit, including the maximized log likelihood function value, the system Akaike's information criterion (AIC) value, and the system $R^{2}$ value. Each of the estimated structural change models has an identical number of free parameters (31). And each is, of course, non-nested with respect to the other. Therefore, in this case if we simply follow the advice of Pollak and Wales (1991), which, based on the likelihood dominance criterion, or LDC, we choose the quadratic structural change model, or the IAIDS model based on the exponential transition function in (11), as the preferred specification among the three structural change models considered..$^{5}$

It is useful to obtain insights into the nature of the implied structural change. The underlying time paths of structural change generated from the estimated transition function for each of the three models are reported in the top panel of Figure 1. While each model suggests a somewhat different adjustment path - the first-order model, for example, implies something not far off from linear structural change-it is of interest that in no case is structural change a discrete phenomenon. ${ }^{6}$ The preferred model, that is, the second-order model, implies that structural change in meat demand is symmetric around the third quarter of 1972 .

Additional insights into the nature of the structural change implied by the preferred model 
can be obtained by examining the implied own-price and scale flexibilities over time, and by comparing these values with those obtained for the basic IAIDS model. Here we compute mean paths for these flexibilities at every data point by using 1000 bootstraps of each model. To aid in the interpretation we also compute empirical 90-percent confidence intervals for the mean paths associated with the basic IAIDS. The results are reported in Figure 2, where the panels in the left-hand column correspond to the own-price flexibilities for beef, pork, and poultry, respectively, and those in the right-hand column are the corresponding scale flexibilities. There are several notable features depicted in Figure 2. To begin, the structural change implied by the second-order model results in mean paths for the beef own-price flexibility that are mostly within the 90-percent confidence bands for the basic model. This is not the case for pork and poultry, with the own-price flexibilities for pork implied by the second-order model seldom being within the 90-percent confidence bands. Most striking, however, are the patterns revealed for the scale flexibilities. The panels in the right-hand column of Figure 2 show that scale flexibilities implied by the second-order IAIDS depart significantly from those for the basic IAIDS over much of the sample period.

\section{Concluding Remarks}

In this paper we have introduced a framework for systematically exploring the nature of structural change in systems of demand equations estimated with time series data. The methodology builds on the time varying regression models explored by Lin and Teräsvirta (1994), and considers the possibility that structural change is continuous and non-monotonic. An application is considered in the context of inverse demand equations for beef, pork, and poultry. The results show that the best fitting model is associated with structural change that has been non-monotonic over time. The implications for estimated own-price and scale flexibilities are found to be non-trivial. 


\section{Notes}

*Matthew Holt is Professor and Wickersham Chair of Excellence in Agricultural Research and Joseph Balagtas is Assistant Professor, Department of Agricultural Economics, Purdue University. Invited Paper, 2009 AAEA Annual Meetings, July $26^{t h}-28^{t h}$, Milwaukee, WI.

${ }^{1}$ Goodwin and Brester (1995) and more specifically, Goodwin et al. (2003), use a methodology that is similar to what we propose here in the context of demand system estimation. Even so, in neither case did the authors consider the possibility that structural change was non-monotonic over time.

${ }^{2}$ Because adding-up must hold at all data points, it follows that $\sum_{i=1}^{n} e_{i t}=0$ for all $t$, implying that the corresponding $n x n$ covariance matrix is rank deficient. As Barten (1969) shows, however, FIML estimates may be obtained by arbitrarily deleting an equation and by using an iterative Seemingly Unrelated Regression (SUR) estimation strategy.

${ }^{3}$ Of course the IAIDS model that utilizes the Stone quantity index in lieu of the tranlog quantity index $\ln (Q)$, the so called linearized IAIDS, is in fact linear in parameters. A future research agenda might therefore be to incorporate the Taylor approximation approach for testing for structural change advocated by Luukkonen et al. (1988) and Lin and Teräsvirta (1994) into the linearized IAIDS.

${ }^{4}$ Preliminary estimates were also conducted by imposing unit roots on the autoregressive structure. The results obtained in this case regarding structural change are comparable to those that we report here. We continue to use Holt's (1998) autocorrelation correction methods in all subsequent analysis, however, in that formal tests for unit roots in the context of the (nonlinear) IAIDS model are not well defined. This remains as an important area for future research.

${ }^{5}$ Additional bootstrap tests were performed to determine if structural change is a feature of, collectively, the constant terms, the constant and dummy variable terms, and the constant and structural IAIDS parameters. In all cases the null model of no structural change was rejected at the 0.001 level. As well, bootstrap tests were performed using the second-order IAIDS as the base model to determine if the autocorrelation parameters remained stable over time. The empirical $p$-value in this case is 0.377 , implying that the autocorrelation process is reasonably stable over time.

${ }^{6}$ It is not possible for the second-order model to provide an approximation to discrete structural change. In the first- and third-order models, however, estimated values for $\gamma$ that are suitably large will result in a model that effectively has discrete breaks. 


\section{References}

Alston, J. M. and J. A. Chalfant (1991). Can we take the con out of meat demand studies? Western Journal of Agricultural Economics 16, 36-48.

Andrews, D. W. K. (1993). Tests for parameter instability and structural change with unknown change point. Econometrica 61(4), 821-856.

Andrews, D. W. K. and W. Ploberger (1994). Optimal tests when a nuisance parameter is present only under the alternative. Econometrica 62 (6), 1383-1414.

Barten, A. P. (1969). Maximum likelihood estimatiion of a complete system of demand equations. Europen Economic Review 1, 7-63.

Barten, A. P. and L. J. Bettendorf (1989). Price formation of fish : An application of an inverse demand system. European Economic Review 33(8), 1509 -1525.

Bryant, H. L. and G. C. Davis (2008). Revisiting aggregate U.S. meat demand with a bayesian averaging of classical estimates approach: Do we need a more general theory? American Journal of Agricultural Economics 90(1), 103-116.

Chavas, J.-P. (1983). Structural change in the demand for meat. American Journal of Agricultural Economics 65(1), 148-153.

Chow, G. C. (1960). Tests of equality between sets of coefficients in two linear regressions. Econometrica 28(3), 591-605.

Dahlgran, R. A. (1987). Complete flexibility systems and the stationarity of U.S. meat demand. Western Journal of Agricultural Economics 12, 152-163.

Davies, R. B. (1977). Hypothesis testing when a nuisance parameter is present only under the alternative. Biometrika 64(2), 247-254.

Davies, R. B. (1987). Hypothesis testing when a nuisance parameter is present only under the alternatives. Biometrika $74(1), 33-43$.

Davis, G. C. (1997). The logic of testing structural change in meat demand: A methodological analysis and appraisal. American Journal of Agricultural Economics 79(4), 1186-1192.

Deaton, A. and J. Muellbauer (1980). An Almost Ideal Demand System. American Economic Review $70(3), 312-326$.

Eales, J. S. and L. J. Unnevehr (1988). Demand for beef and chicken products: Separability and structural change. American Journal of Agricultural Economics 70 (3), 521-532.

Eales, J. S. and L. J. Unnevehr (1994). The inverse almost ideal demand system. European Economic Review 38(1), $101-115$. 
Farley, J. U., M. Hinich, and T. W. McGuire (1975). Some comparisons of tests for a shift in the slopes of a multivariate linear time series model. Journal of Econometrics 3(3), $297-318$.

Goodwin, B. K. and G. W. Brester (1995). Structural change in factor demand relationships in the U.S. food and kindred products industry. American Journal of Agricultural Economics $77(1), 69-79$.

Goodwin, B. K., D. Harper, and R. Schnepf (2003). Short run demand relationships in the U.S. fats and oils complex. Journal of Agricultural and Applied Economics 35(1), 171-184.

Hansen, B. E. (1996). Inference when a nuisance parameter is not identified under the null hypothesis. Econometrica 64(2), 413-430.

Holt, M. T. (1998). Autocorrelation specification in singular equation systems: A further look. Economics Letters 58, 135-141.

Holt, M. T. (2002). Inverse demand systems and choice of functional form. European Economic Review 46, 117-142.

Holt, M. T. and B. K. Goodwin (1996). Generalized habit formation in an inverse almost ideal demand system: An application to meat expenditures in the U.S. Empirical Economics 78(5), 1396-1396.

Lin, J. C.-F. and T. Teräsvirta (1994). Testing for the constancy of regression parameters against continuous structural change. Journal of Econometrics 62(2), 211-228.

Luukkonen, R., P. Saikkonen, and T. Tersvirta (1988). Testing linearity against smooth transition autoregressive models. Biometrika 75 (3), 491-499.

Moschini, G. and K. D. Mielke (1989). Modelling the pattern of structural change in U.S. meat demand. American Journal of Agricultural Economics 71, 253-261.

Pollak, R. A. and T. J. Wales (1991). The likelihood dominance criterion : A new approach to model selection. Journal of Econometrics 47(2-3), 227-242.

Seo, M. (2006). Bootstrap testing for the null of no cointegration in a threshold vector error correction model. Journal of Econometrics 134(1), 129 - 150.

Skalin, J. (1998, December 13). Testing linearity against smooth transition autoregresison using a parametric bootstrap. Stochkholm School of Economics Working Paper Series in Economics and Finance No. 276.

Terasvirta, T. (1994). Specification, estimation, and evaluation of smooth transition autoregressive models. Journal of the American Statistical Association 89(425), 208-218.

U.S. Department of Agriculture (2006a, August). Economic resaerch service. poultry yearbook. Online. Stock No. 89007.

U.S. Department of Agriculture (2006b, January). Economic research service. red meat yearbook. Online Publication. Stock No. 94006. 
Table 1: Key Measures of Model Fit and Select Parameter Estimates for Basic and Time-Varying Meat Demand Models.

\begin{tabular}{|c|c|c|c|c|}
\hline & $\underline{\text { Basic IAIDS }}$ & $\underline{\text { First Order }}$ & $\underline{\text { Second Order }}$ & $\underline{\text { Third Order }}$ \\
\hline No. of Parameters & 16 & 31 & 31 & 31 \\
\hline Log Likelihood & 1388.31 & 1416.61 & 1420.01 & 1417.90 \\
\hline System AIC & -21.009 & -21.157 & -21.195 & -21.172 \\
\hline System $R^{2}$ & 0.9992 & 0.9994 & 0.9994 & 0.9994 \\
\hline$\left|\Sigma_{T V}\right| /\left|\Sigma_{L}\right|$ & - & 0.729 & 0.702 & 0.718 \\
\hline LR Test Statistic & & 56.599 & 63.417 & 59.192 \\
\hline Bootstrapped $P$-value & - & $<0.001$ & $<0.001$ & $<0.001$ \\
\hline Curvature Violations & 52 & 0 & 3 & 0 \\
\hline$\gamma$ & - & $\begin{array}{c}1.281 \\
(0.733)\end{array}$ & $\begin{array}{c}1.070 \\
(0.326)\end{array}$ & $\begin{array}{c}1.165 \\
(0.480)\end{array}$ \\
\hline $\mathrm{c}$ & - & $\begin{array}{c}0.376 \\
(0.183)\end{array}$ & $\begin{array}{c}0.285 \\
(0.027)\end{array}$ & $\begin{array}{c}0.677 \\
(0.048)\end{array}$ \\
\hline
\end{tabular}

Note: System AIC denotes the system Akaike information criterion. $\left|\Sigma_{T V}\right| /\left|\Sigma_{L}\right|$ denotes the ratio of the determinant of the error covariance matrix for the basic IAIDS model relative to that for the respective time-varying IAIDS model. The row titled 'Bootstrapped $P$-value' denotes the empirical $p$-value for the LR test of the respective structural change model relative to the base model obtained from 999 parametric bootstrap replications. Values in parenthesis are asymptotic standard errors. There are 180 sample observations. 


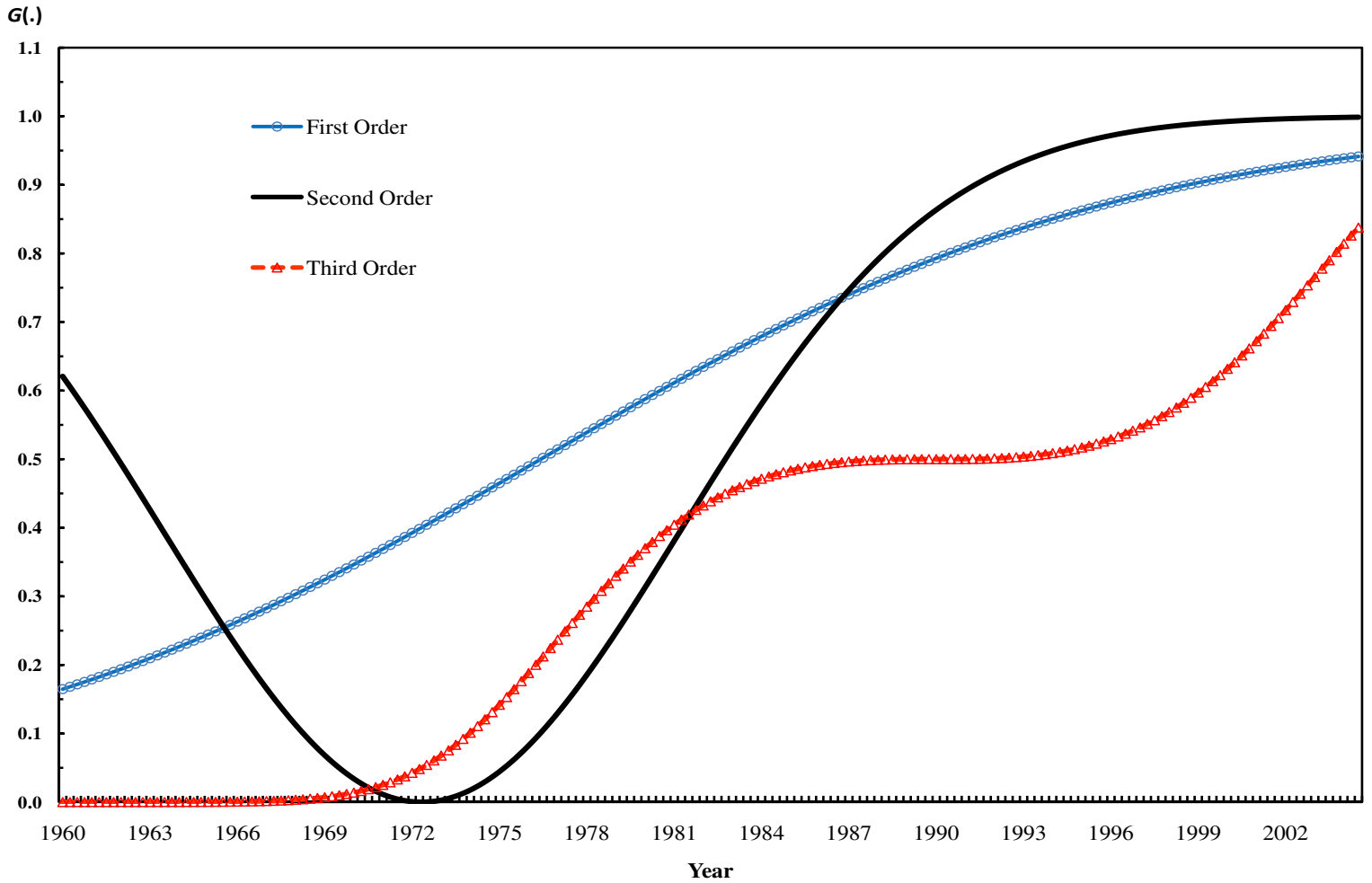

Panel (a)

Figure 1: Estimated Transition Functions for First-Order, Second-Order, and Third-Order Structural Change IAIDS Models. 

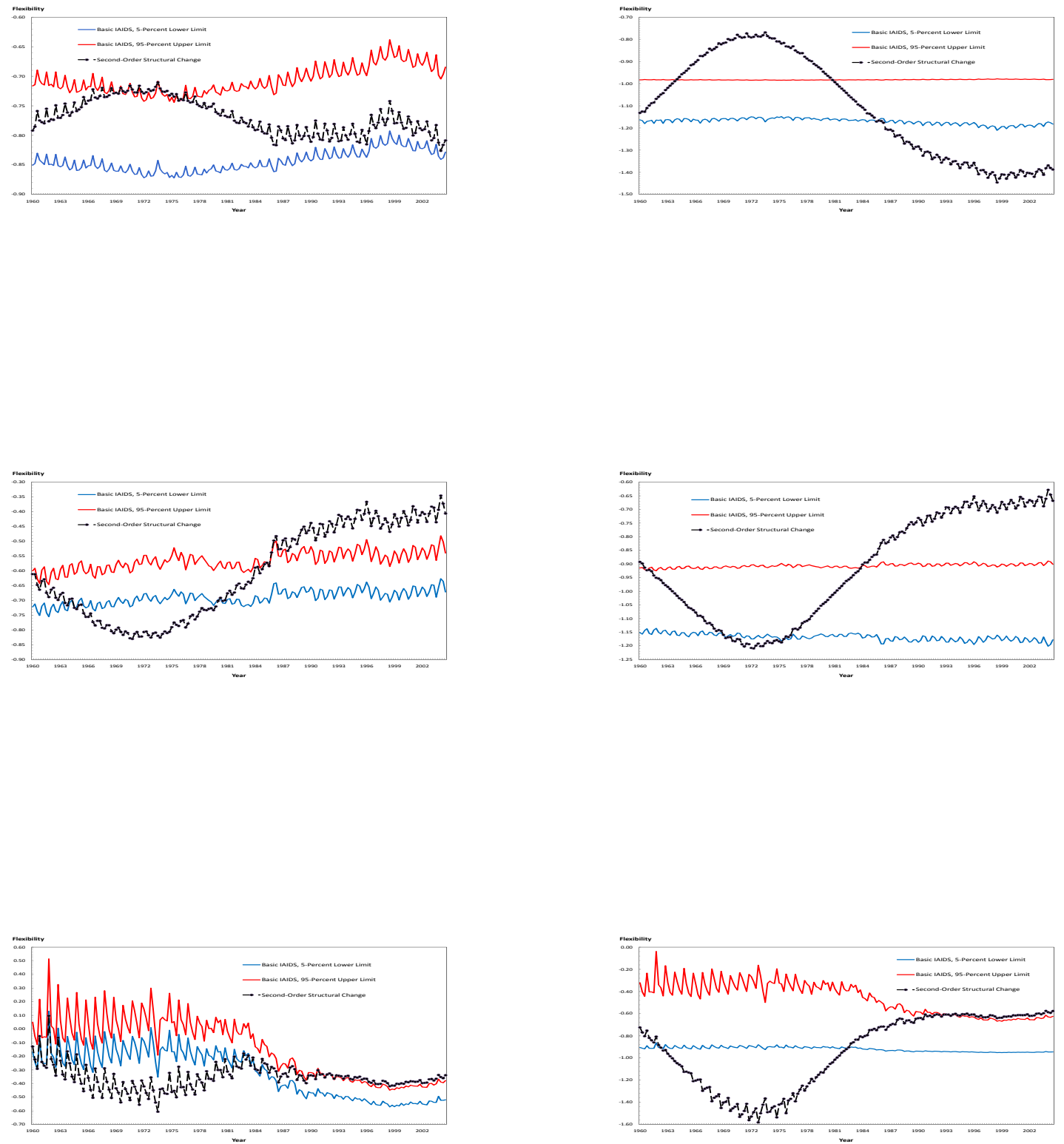

Figure 2: Time-Series Plots of 90-Percent Flexibility Confidence Bands for the Basic IAIDS and Mean Paths for Flexibilities for the Second-Order Structural Change IAIDS. (a) Beef, Own-Price Flexibility; (b) Beef, Scale Flexibility; (c) Pork, Own-Price Flexibility; (d) Pork, Scale Flexibility; (e) Poultry, Own-Price Flexibility; and (f) Poultry, Scale Flexibility 